\title{
In Vivo Mapping of Cortical Columnar Networks in the Monkey with Focal Electrical and Optical Stimulation
}

\author{
Anna Wang Roe ${ }^{1 *}$, Mykyta M. Chernov ${ }^{2}$, Robert M. Friedman ${ }^{2}$ and Gang Chen ${ }^{1}$ \\ ${ }^{1}$ Interdisciplinary Institute of Neuroscience and Technology, Zhejiang University, Hangzhou, China, ${ }^{2}$ Department of \\ Psychology, Vanderbilt University, Nashville, TN, USA
}

There are currently largescale efforts to understand the brain as a connection machine. However, there has been little emphasis on understanding connection patterns between functionally specific cortical columns. Here, we review development and application of focal electrical and optical stimulation methods combined with optical imaging and fMRI mapping in the non-human primate. These new approaches, when applied systematically on a large scale, will elucidate functionally specific intra-areal and interareal network connection patterns. Such functionally specific network data can provide accurate views of brain network topology.

Keywords: cortical column, optogenetics, microstimulation, fMRI, monkey, optical imaging, optical stimulation, infrared neural stimulation

\section{OPEN ACCESS}

Edited by:

Kathleen S. Rockland, Boston University School of Medicine,

USA

Reviewed by:

Zoltan F. Kisvarday,

University of Debrecen, Hungary

Wim Vanduffel,

Harvard Medical School, USA

*Correspondence:

Anna Wang Roe

annawang@zju.edu.cn

Received: 19 June 2015 Accepted: 12 October 2015 Published: 16 November 2015

Citation:

Roe AW, Chernov MM, Friedman RM and Chen G (2015) In Vivo Mapping of Cortical Columnar Networks in the Monkey with Focal Electrical and Optical Stimulation.

Front. Neuroanat. 9:135. doi: 10.3389/fnana.2015.00135

\section{A VIEW OF THE PRIMATE BRAIN}

In primates (both human and non-human), much of brain volume (up to 80\%) is occupied by connections between different parts of the cerebral cortex. Specificity of these connections forms information processing networks that are critical to normal sensory, motor, and cognitive function. Interruption of these connections (e.g., by trauma, stroke) leads to loss or alteration of function. In this sense, the brain can be viewed as a connection machine. There are now largescale projects (termed connectome projects) underway worldwide to study this connection machine (e.g., www.humanconnectomeproject.org, www.brainnetome.org, www.mouseconnectome.org). However, one aspect of these connectome studies that has largely been neglected is the fact that in humans and in primates, cerebral cortex is composed of basic functional columnar units. These units are on the order of a few hundred microns in size and have specific functions (e.g., in visual cortex: processing visual color, shape, depth, or motion information) and are connected in networks with other cortical columns of similar or related functionality (Figure 1). Such columnar networks form a basic feature of primate brain architecture. Thus, inherent in understanding the connection machine in primates is developing the ability to systematically map connection patterns between sets of cortical columns.

Current human connectomes (based on fMRI study) lack the spatial resolution for examining connection patterns at the columnar scale and therefore lack functional specificity inherent in columnar organization. Individual voxels in these studies represent averages of multiple functional columns (Figure 2, yellow box represents $3 \mathrm{~mm}$ voxel, white box represents $1 \mathrm{~mm}$ voxel); networks of voxels (Figure 2, yellow and white bidirectional arrows) therefore represent connections between averages of multiple functional networks. Due to the differential connectivity patterns of individual columns (e.g., color blobs to thin stripes vs. orientation columns to pale/thick 

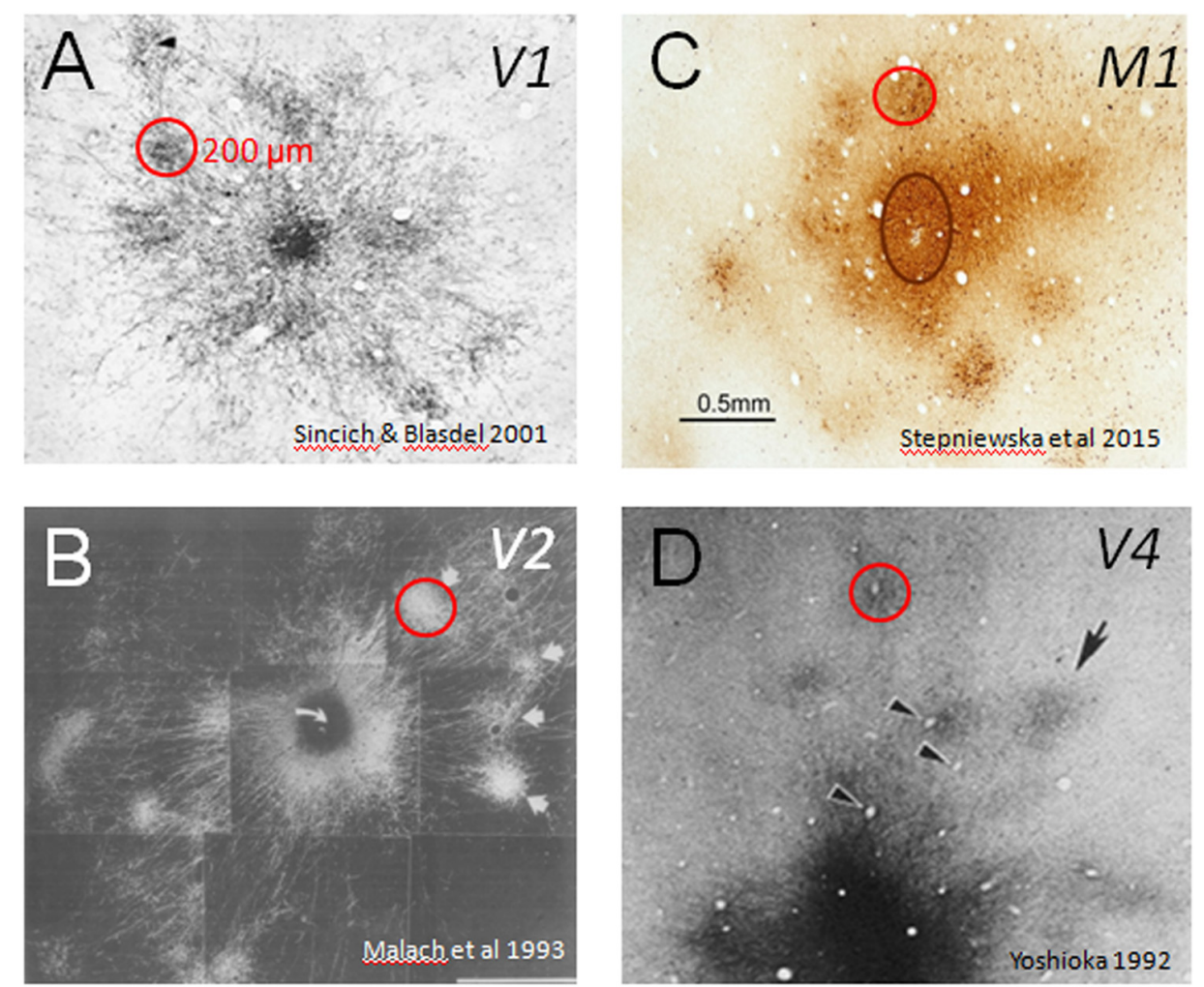

FIGURE 1 | Cortical modules are connected in specific networks. Examples of connected columns in (A) V1 (Sincich and Blasdel, 2001 ), (B) V2 (Malach et al., 1993), (C) M1 (Stepniewska et al., 2015), and (D) V4 (Yoshioka et al., 1992). Red circles indicate that column sizes, roughly $200 \mu \mathrm{m}$ in size, are common to many cortical areas. Scale bar: $0.5 \mathrm{~mm}$ applies to all.

stripes, Livingstone and Hubel, 1984; Roe and Ts'o, 1999, 2015; Sincich et al., 2010; Federer et al., 2013), such averages can lead to inaccurate and misleading conclusions about cortical networks.

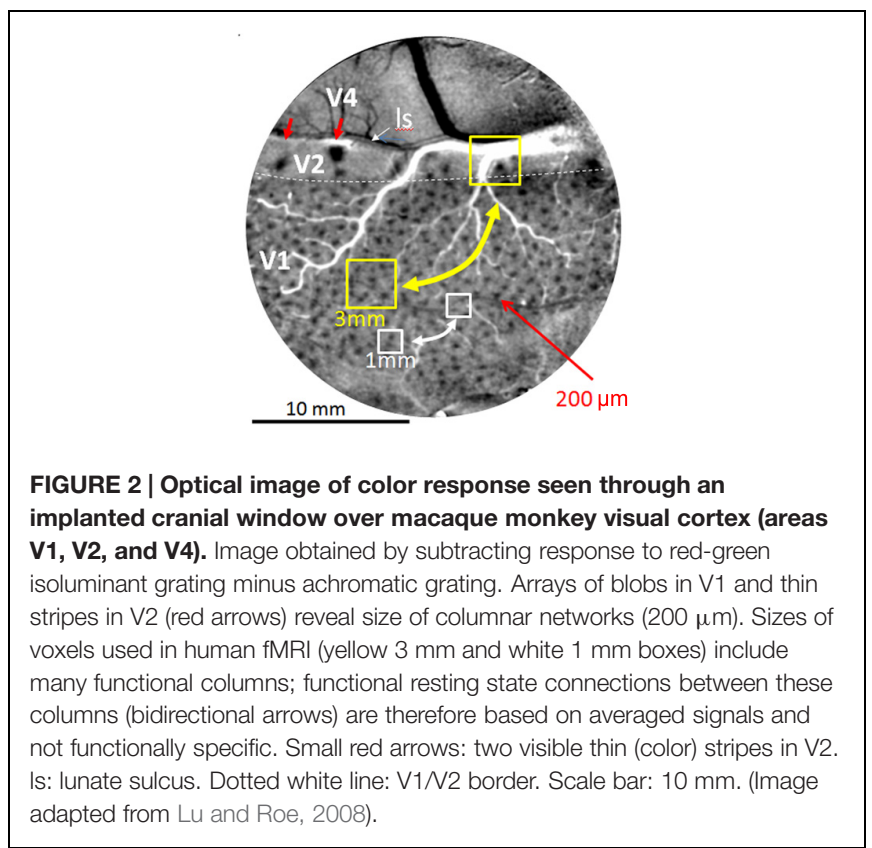

\section{Note on 'Column' Terminology}

Cortical functional units have been described in many areas of visual cortex (V1, V2, V3, V4, MT, inferotemporal cortex) as well as other sensory, parietal, and prefrontal areas and have been termed columns, puffs, blobs, clusters, modules, and domains. Although each term is based on slightly different functional and/or anatomical criteria, they all capture the concept of modularity. For purposes of this review, we will use the term 'column', as it is a generally familiar term and portrays the concepts of: 'small' (200-300 $\mu \mathrm{m}$ in size), 'repeated' (general architectural unit of the cortex), and 'functional' (composed of cluster of neurons which share similar function). This review proposes ways to examine the 'columnar connectome.'

Note that the concept of a column has of late been controversial, partly because there is not a single accepted definition and because by some criteria cortex is not clearly columnar (e.g., see Rockland, 2010). We use the term 'column' less as a physical construct (as its precise nature still needs further study) and more as a term to refer to a modular unit. Our concept of the 'column' is that it is a unique site of integration in cerebral cortex, one which is defined by a unique set of (often patchy) inputs and outputs. It is these inputs and outputs that result in a population response that is shared among a cluster of neurons. Although there is controversy regarding what exactly defines the column, the ability to visualize modules of 
functionally distinct preference is a strong statement that there is modular organization.

\section{A COLUMNAR MONKEY CONNECTOME: A PROPOSAL}

We propose to pursue a columnar connectome in the monkey; such a connectome would provide a functionally specific understanding of brain networks. While attaining the spatial resolution needed to see columns in humans may be on the horizon (Cheng et al., 2001; Yacoub et al., 2008; Sun et al., 2013), the ability to do so in monkeys is already at hand. Functional imaging at high magnetic fields coupled with use of surface coils has enabled submillimeter resolution in anesthetized squirrel monkeys (Chen et al., 2007), and in awake macaque monkeys can sufficiently distinguish supra-, middle-, and infra-granular laminar specificity and fingerprint V1, V2, and V4 laminar profiles (Chen et al., 2012a,b, 2013; Goense et al., 2012; see also, Olman et al., 2012; Shih et al., 2013; Baek et al., 2015). Moreover, monkeys are excellent animal models for human behavior and disease. They share many commonalities with man including common behavioral repertoires and similar brain structures including the columnar nature of cortical organization (Table 1). Furthermore, monkeys can undergo experimental manipulations to answer questions in ways that are not possible in humans. Note that while mouse connectomes have decided advantages (e.g., genetic manipulability), mouse cerebral cortex (except for barrel cortex) is not columnar in the same way as primate cortex. For example, visual orientation selective neurons are not organized in columnar fashion (Metin et al., 1988; Schuett et al., 2002). These differences make certain comparisons less valid for understanding human brain structure, function, and behavior (Table 1). A columnar based understanding, we predict, would provide a fundamental component for developing a connectional theory of brain function, one that is not diluted by inaccurate averaged information.

\section{NEED FOR LARGESCALE, FOCAL TRACING METHODS}

Anatomical connections can be conducted at columnar scale and have provided some breathtaking views of cortical connection patterns. Perhaps some of the best examples come from monkey V1 where intra-areal patterns of connections have been well documented (e.g., Ts'o et al., 1986; Bosking et al., 1997). There are also a small number of studies on inter-areal columnar connectivity (Livingstone and Hubel, 1984; Roe and Ts'o, 1999; Shmuel et al., 2005; Federer et al., 2013). However, anatomical studies require sacrifice of the animal, and time consuming histological processing and tracer label reconstruction. Within a single animal, connection patterns from only a handful (about five, cf. Zingg et al., 2014) of distinct sites can be examined without compromising the certainty of label identification, placing a limit on the number of different networks one can study in a single brain.

Other methods for studying connections have yet to achieve columnar resolution. In vivo diffusion imaging is limited in spatial resolution and therefore accuracy. While it has been useful for some tracts (e.g., Jbabdi et al., 2013), a complete mapping of fiber connections at columnar resolution is unlikely (Thomas et al., 2014). Challenges such as identifying correct fiber courses at points of fiber intersection are still being addressed. Functional and anatomical tract tracing methods in humans using electrical stimulation and imaging (e.g., Lujan et al., 2013) or TMS and imaging (Ruff et al., 2009; Driver et al., 2010; Krieg et al., 2013; Johnen et al., 2015) have been informative, but are also low in spatial resolution. Resting state connectivity in humans (for review, Raichle, 2015), a method based on covariation of hemodynamic response between brain sites, also lacks the spatial resolution for revealing columnar connectivity. While high spatial resolution (mm-scale) resting state connectivity in monkeys can be achieved at high fields (Wang et al., 2013), columnar resolution has yet to be achieved.

Given the constraints of current methods, we have therefore sought to develop new methods to elucidate brain networks systematically, at high spatial resolution, and in a high-throughput manner. In the past few years, we have focused on developing in vivo functional tract tracing methods comprised of focal columnar stimulation coupled with optical or fMRI imaging, a method we view as a shortcut toward examining anatomical connectivity. A distinct advantage is that in vivo functional tract tracing does not require histological processing and therefore can be conducted repeatedly in vivo in the same animal. This enables the study of networks, both at the local and the global scale,

TABLE 1 | Comparison of behavioral repertoires and cerebral cortical organization in mouse, monkey, and man.

\begin{tabular}{llll}
\hline & Mouse & Monkey & Man \\
\hline Vision & & & \\
Fovea, high acuity & No & Yes & Yes \\
Color vision & No & Yes & Yes \\
Face perception & No & Yes & Yes \\
Eye movements & No & Yes & Yes \\
Visual attention & No & Yes & Yes \\
Manual behavior & & & \\
Manual and digit behavior & No & Yes & Yes \\
Texture on skin & No & Yes & Yes \\
Limb kinematics & No & Yes & Yes \\
Shape perception via grasp & No & Yes & Yes \\
Visuomotor directed grasp & No & Yes & Yes \\
Social behavior & & & \\
Language/response alternation & No & Yes & Yes \\
Social complexity & No & Yes & Yes \\
Brain organization & & & Yes \\
Orientation columns & No & No & Yes \\
Large cortex & & &
\end{tabular}


from multiple stimulation sites, enabling systematic and large scale collection of connectivity data from a single animal. It is hoped that study of multiple connection networks within single animals can more easily provide data useful for graph theoretical analysis and characterization of network topologies (cf. Sporns, 2010). Note that functional tract tracing is not meant to replace anatomical studies of brain connections, but rather to complement and extend our understanding of the anatomical gold standard.

\section{THREE FUNCTIONAL TRACT TRACING METHODS}

In the following sections, we will describe three functional tract tracing methods that could be used in a high-throughput manner: electrical, optogenetic, and near infrared laser stimulation. Each has its strengths and weaknesses and may be chosen depending on the questions at hand.

\section{Focal Electrical Stimulation}

Electrical stimulation has been a long-standing tool for functional mapping of the brain (Rasmussen and Penfield, 1947). Its uses have spanned mapping cortical organization, distinguishing functional relationships between cortical areas, and applications in brain-machine interfaces (Tolias et al., 2005; Moeller et al., 2008; Stepniewska et al., 2009, 2011; Chase et al., 2012). Importantly, electrical stimulation has been shown to generate or alter normal percepts and behaviors in many species, including human and non-human primates (Salzman et al., 1990; Romo et al., 2000; Godde et al., 2002; Graziano et al., 2002; Murphey and Maunsell, 2007; Tehovnik and Slocum, 2009). However, the relationship between circuits and behaviors activated by electrical stimulation remains poorly understood. This is partially due to a lack of a good understanding of the intra-areal and inter-areal networks activated or modulated via intracortical microstimulation (Bullmore and Sporns, 2009). The power of combining electrical stimulation with functional imaging in a behavioral context has been pioneered in monkeys by Ekstrom et al. (2008, 2009). By conducting fMRI concurrently with stimulation of FEF in the macaque, they demonstrated a contrastdependent enhancement of visual cortical processing, thereby linking visual behavioral effects with underlying anatomical networks. Developing this approach at columnar resolution would further our understanding of both local and global circuits underlying behavior.

Toward this goal, we have developed methods to map intraareal and inter-areal circuits using optical imaging combined with focal microstimulation. The underlying viewpoint is that stimulation of a single functional column will lead to activation of other connected columns, both intra-areal and inter-areal. Although electrical stimulation is accompanied by current spread, when delivered at appropriate levels, the effect can remain relatively local. In a two photon study of local neuronal activation in response to well-controlled, low current (5-10 $\mu \mathrm{A})$ electrical microstimulation, Histed et al. (2009) demonstrated that electrically activated neurons comprised a subset of the neurons within the local vicinity $(300 \mu \mathrm{m})$ and that movement of the stimulating electrode by $10^{\prime} \mathrm{s}$ of microns resulted in activation of different subsets of neurons within the same local vicinity. This suggested that, with the right stimulation parameters and precise localization of stimulation location, microstimulation can have quite selective and focal effects.

This finding by Histed et al. (2009) suggested the feasibility of using electrical microstimulation to map local columnar networks. Encouraged by this study, we attempted functional mapping with electrical microstimulation in monkey somatosensory cortex. As electrical stimulation can produce either excitatory or suppressive effects depending on the stimulation intensity, it was vitally important to characterize stimulus amplitudes by systematically characterizing the stimulation parameter space (e.g., intensity, duration, laminar location, cf. Tehovnik et al., 2004, 2006, 2009). Using trains of biphasic pulses (typically $200 \mathrm{~Hz}, 0.4 \mathrm{~ms}$ pulse duration), and varying intensity by testing different current amplitudes (10-300 $\mu \mathrm{A})$ and pulse numbers $(1,13,26,63$ pulses, resulting in total train durations of $0.4,50,100,250 \mathrm{~ms}$ ), we examined the effect of stimulation intensity on neuronal firing and hemodynamic signal as measured with intrinsic signal optical imaging. With chosen electrical stimulation parameters $(25 \mu \mathrm{A}$, $250 \mathrm{~Hz}, 100 \mathrm{~ms}$ ), the imaged hemodynamic response to electrical stimulation mimicked that of tactile stimulation (Figure 3C);

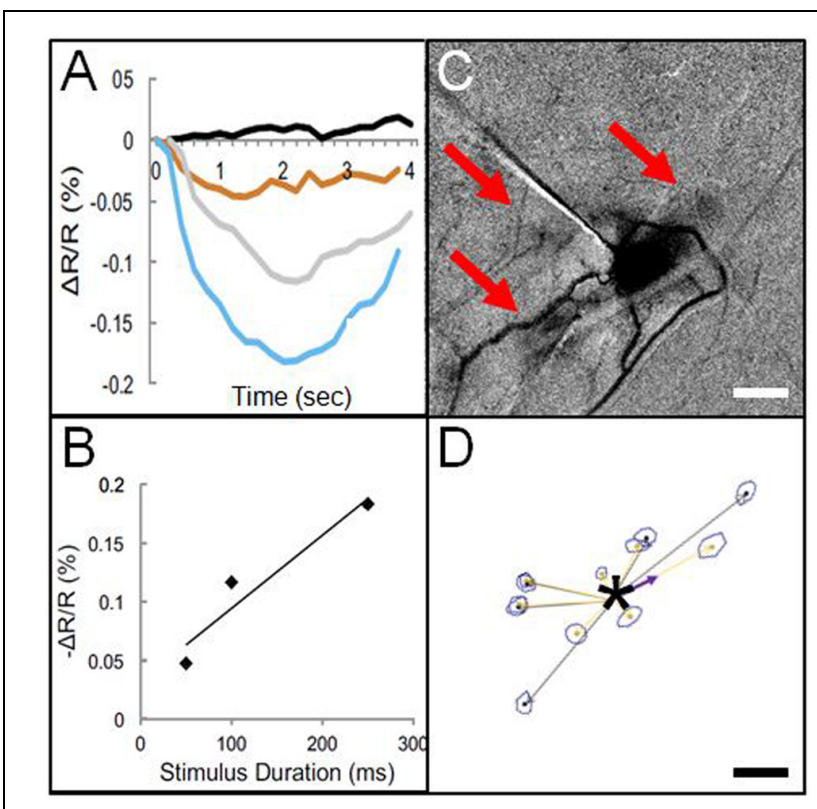

FIGURE 3 | Optical imaging of focal electrical stimulation in squirrel monkey somatosensory cortex. (A) Stimulation at $0 \mathrm{~s}$ (black), $50 \mathrm{~ms}$ (orange), $100 \mathrm{~ms}$ (gray), and $250 \mathrm{~ms}$ (blue) at $150 \mu \mathrm{A}$. Y axis: reflectance change $d R / R$. (B) Optical reflectance change increases with stimulus duration (measured at site of stimulation). (C) Optical image of local functional connectivity (from Brock et al., 2013) appears similar to (D) patches of anatomical label following focal (250 $\mu \mathrm{m}$ sized) injection (asterisk) of BDA tracer (adapted from Negyessy et al., 2013). (C,D) are from different animals. Scale bar for $(\mathbf{C}, \mathbf{D}): 1 \mathrm{~mm}$. 
both revealed a focal $1 \mathrm{~mm}$ activation site consistent with a single digit representation. Increasing intensity of stimulation [either current amplitude (Figure 3A) or number of pulses, (Figure 3B)] led to increasing magnitudes of imaged reflectance change. Importantly, stimulation (e.g., $200 \mu \mathrm{A}$ for $250 \mathrm{~ms}$ ) elicited not only a focal activation at the site of stimulation but other focal activations within 1-2 $\mathrm{mm}$ of the stimulated site (Figure 3C). This activation pattern is very similar in appearance to typical intra-areal columnar networks revealed by anatomical tracer studies (Figures 1 and 3D). Direct confirmation of this anatomical correspondence is currently in progress.

There is also potential for this approach to be used for mapping inter-areal columnar connections. Such an approach has been used to examine connections between motor and parietal areas in the prosimian primate the Galago (Stepniewska et al., 2011). Previous studies have shown that stimulation of parietal cortex at selected sites produces classes of complex behaviors (such as defensive postures, feeding, grooming; Cooke et al., 2003; Stepniewska et al., 2009). To understand the anatomical networks underlying these motor behaviors, optical imaging was used to map motor cortex during stimulation of behaviorally characterized parietal sites. Activations in motor cortex following parietal stimulation were intensity-dependent (Figure 4A) and revealed site-dependent differential topography (Figures 4B,C). In another study, Arsenault et al. (2014) mapped the functional consequences of stimulating ventral tegmental area with fMRI as well evaluating its behavioral effects and found that free choice behavior could be dramatically altered via widespread activation of the dopaminergic reward system. These studies suggest the possibility of simultaneously evoking or modulating a behavioral effect and mapping the underlying inter-areal circuit.

\section{Focal Optical Stimulation \\ Advantages}

2015 has been designated by the United Nations as the International Year of Light. We owe this to pioneers such as Nobel Laureates in Physics 2009 Charles Kao, Willard Boyle, and George Smith who were innovators in the development of fiber optics and CCD chips. These technologies enabled many advances in optical engineering and in medicine, including technologies for brain stimulation. There are several advantages to replacing wires with light. The most important advantage of this method over electrical stimulation is that it is not encumbered by current spread, making its effect focal. The volume of affected neural tissue is determined by the light wavelength and size of the delivery fiber optic. By selecting optical fibers with illumination spot sizes of $100 \mu \mathrm{m}$ to $1 \mathrm{~mm}$ in diameter, activation of single to several cortical columns can be achieved (Cayce et al., 2011, 2013). Fiber optics can also be selected for stimulation at the brain surface without direct contact to neural tissue and can be easily targeted to specific cortical locations. Deep tissue stimulation via insertion of fiber optics into deep structures is also possible (e.g., Gerits et al., 2012). Another advantage of using light is that it is not accompanied by electrical stimulation artifact, making it readily compatible with electrical recordings. Also, light is easily applied within magnetic fields, making it useful for functional tracing in the MRI.

\section{Stimulation through Optical Windows}

Optical stimulation techniques can now be introduced via optical windows on the brain. In monkeys, both in anesthetized and awake behaving states, such windows offer the opportunity to probe the brain with multiple techniques. These windows permit multiscale study in individual animals, using methods such as behavioral study, fMRI, optical imaging, electrophysiology, focal stimulation (electrical, optogenetic near infrared laser stimulation), and the study of anatomical connectivity via targeted tracer injections through the window.

\section{Infrared Neural Stimulation (INS)}

The use of infrared wavelength light to evoke neural response was developed by Duco Jansen and colleagues at Vanderbilt University (Wells et al., 2005a,b, 2007a,b). First developed for stimulation of peripheral nerve, this effect is mediated via heat transient induced changes (absorption of infrared light by water) in membrane capacitance and protein conformation (Wells et al., 2007a; Shapiro et al., 2012). The transfer of energy is related to native resonance of water molecules: the better the match between stimulation wavelength and resonant frequency of water, the greater the energy transfer, and therefore, the greater the resulting heat transient; as a corollary, the greater the transfer, the less tissue penetration. Thus, Jansen and colleagues identified a stimulation wavelength $(1.875 \mu \mathrm{m})$ that resulted in reasonable energy transfer and reasonable tissue penetration of 200-300 $\mu \mathrm{ms}$. When this near infrared wavelength light is presented in brief $(0.25 \mathrm{~ms})$ pulses, the transients lead to membrane depolarization and resulting action potentials (see Chernov and Roe, 2014a for review). In contrast to optogenetic approaches, this method is not dependent on viral infection and is thus more amenable to human application; however, unlike optogenetics, this method does not target specific cell types.

\section{Infrared Neural Stimulation Intensity Predicts Magnitude of Cortical Response}

Focal INS stimulation induces neural response as assessed with electrophysiology, optical imaging, and BOLD fMRI imaging. Magnitude of this response is related to the intensity of stimulation (Figures $\mathbf{5 A}-\mathbf{C}$ ). This focal stimulation can elicit functionally specific effects related to columnar organization. In visual cortex, application of focal stimulation to single ocular dominance columns in V1 can, in combination with visual stimulation, enhance response of shared eye ocular dominance columns (Figures 5D,E), suggesting a selective modulation of functionally specific intra-areal networks in V1. Similar modulatory results, by electrical stimulation, have been obtained by Ohayon et al. (2013).

Note that, similar to inhibitory effects of electrical stimulation, optical stimulation can also lead to relative suppression of cortical response. We have observed this using fiber optics of different diameters aimed at cortical columns. We find a U-shaped response where small fibers $(100 \mu \mathrm{m})$ evoke a response weaker 


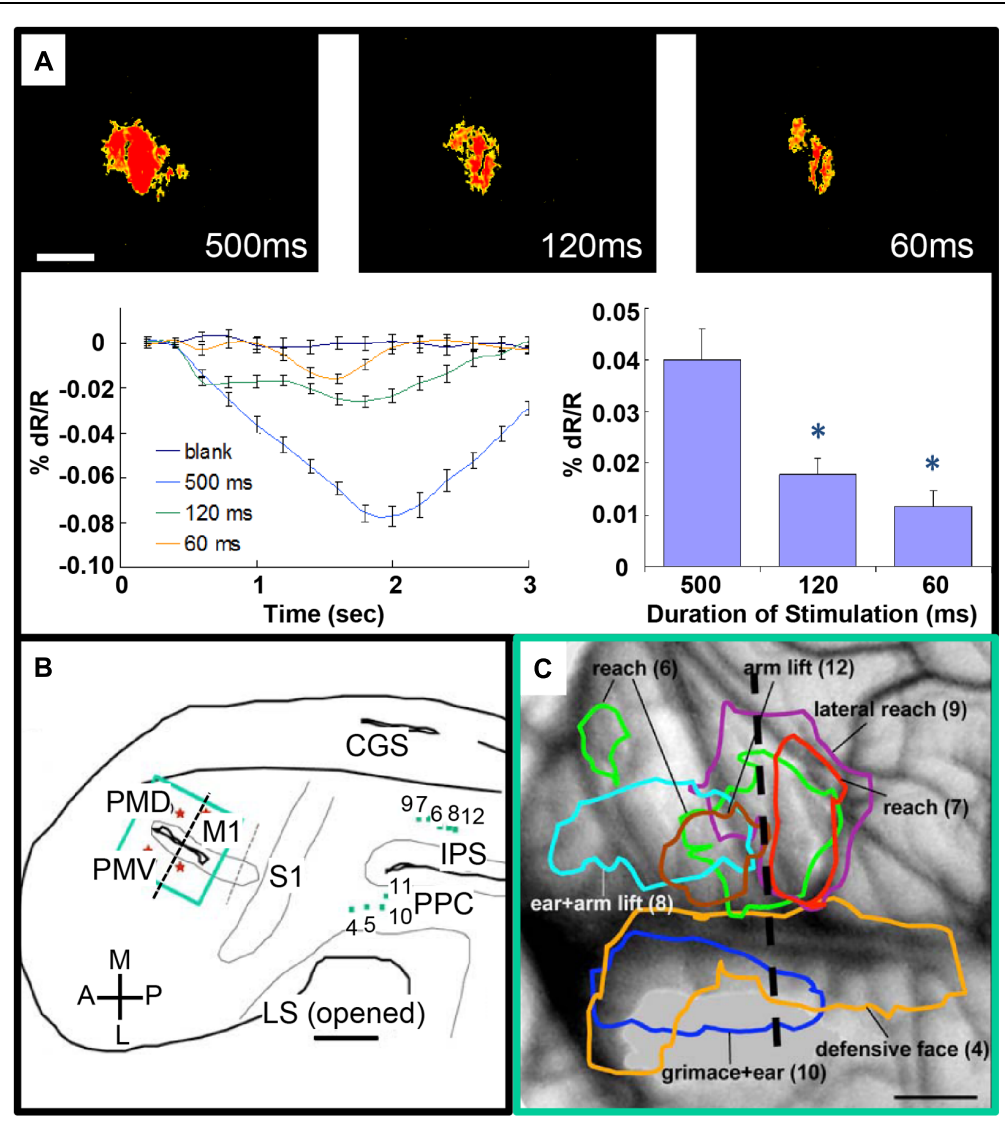

FIGURE 4 | Intrinsic motor cortex activity to different durations of electrical stimulation of PPC. (A) Top: three optical images (only significant pixels shown in red, $t$-test $\left.{ }^{*} p<0.01\right)$ in response to electrical stimulation $(400 \mu \mathrm{A}, 0.1 \mathrm{~ms}$ biphasic, $300 \mathrm{~Hz})$ of the dorsal PPC that evoked a lateral reaching movement (left: 500 ms, middle: 120 ms, right: 60 ms stimulation duration). Below: intrinsic signal time courses (left) and peak magnitudes (right). (B) Reconstruction of hemisphere. Green dots: stimulation sites in PPC. Green rectangle: imaging field of view centered over motor and premotor cortex. (C) Areas of activation in M1, PVD and PMV elicited by electrical stimulation of PPC. Colored outlines: areas activated during face movements (lateral) and forelimb movements (medial). Scale bars: $1 \mathrm{~mm}$. (From Stepniewska et al., 2011).

than $200 \mu \mathrm{m}$ fibers and $400 \mu \mathrm{m}$ or $1 \mathrm{~mm}$ diameter fibers lead to relatively suppressed effects (Figure 5F). We interpret this as the larger fibers recruiting additional inhibitory circuits in the surround which change the balance of ongoing intra-areal networks and result in relative suppression.

\section{Functional Tract Tracing with INS}

Having characterized the effects of INS stimulation, we next examined whether it could be used as a tool for functional tract tracing. To further motivate the use of this approach, we examined the compatibility of INS with fMRI (Chen et al., 2012c). With fMRI, similar to hemodynamic signals measured with optical imaging, the relationship of INS intensity is proportional to hemodynamic BOLD response (Figure 6A). Preliminary studies in squirrel monkeys implanted with optical chambers over somatosensory cortex (imaged with surface coil in a 9.4T Varian magnet) have shown that targeted stimulation of single digit locations (e.g., D2 tip) via apposition of the stimulating fiber optic to the cortical surface at one somatosensory cortical location (Figure 6B) leads to significant BOLD response in topographically appropriate locations in nearby areas (Figure 6C). When imaged in coronal slices, activations appear localized to superficial or middle layers of cortical laminae, suggesting that this resolution is sufficient for superficial, middle, or deep laminar localization (Figure 6C). INS combined with fMRI thus provides the opportunity to examine intra- and inter-areal connection patterns of a stimulated site, as well as identify resulting laminar profiles, that will be useful for interpretation of feedforward or feedback connection patterns.

\section{Behavioral Effects with INS}

There is a long history of focal electrical stimulation producing highly specific behavioral effects (e.g., for motion direction percepts, Salzman et al., 1990; for tactile frequency percepts, Romo et al., 2000). Can INS also be used for similar applications? As a first attempt to examine this possibility, we tested whether focal INS stimulation in visual cortex of macaque monkeys could induce percepts of light spots (phosphenes). In awake fixating monkeys implanted with optical chambers over visual cortex (V1, V2, V4), we used intrinsic signal optical imaging to map the visuotopic representation within the optical chamber (Tanigawa et al., 2010). We then applied 


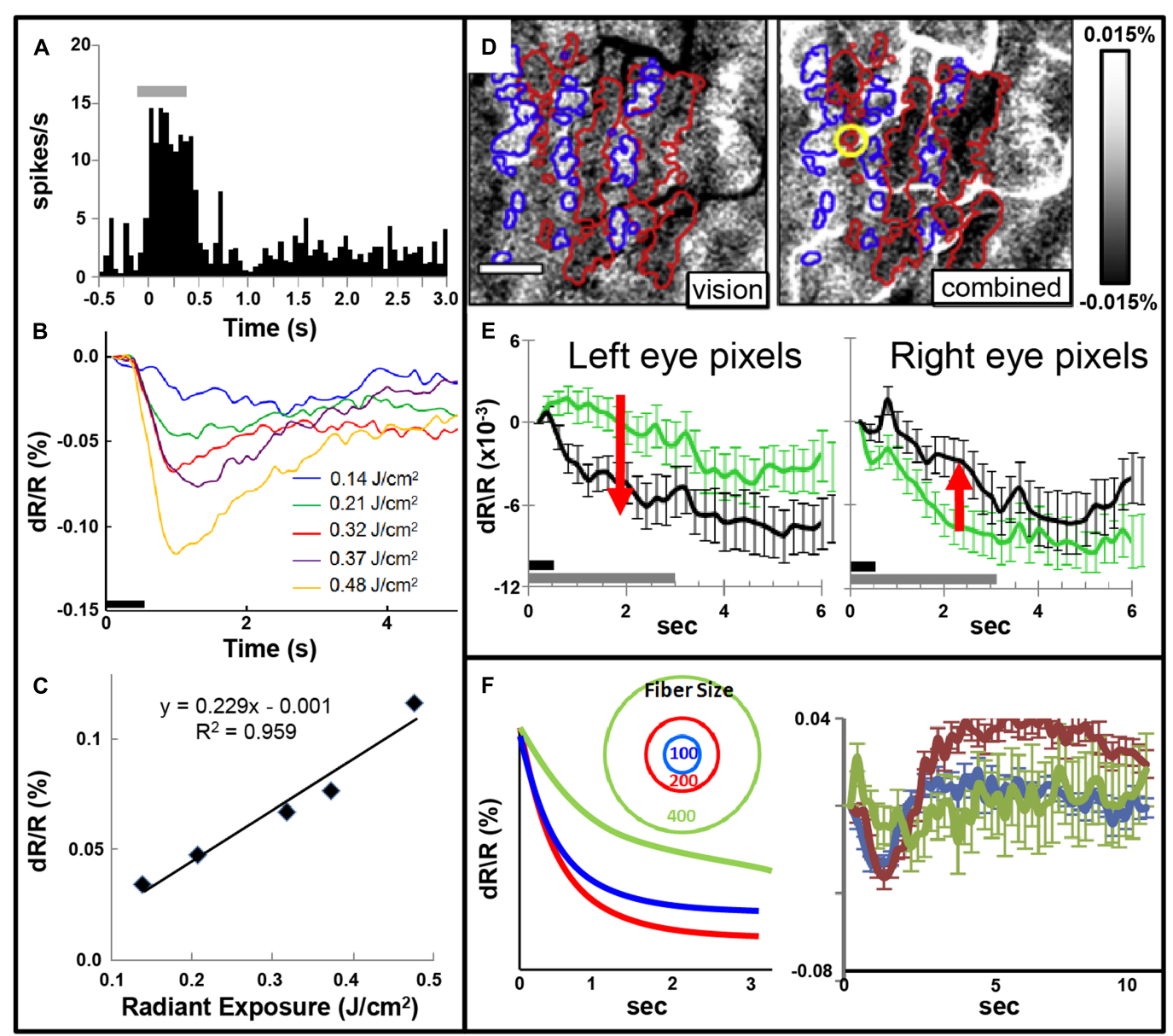

FIGURE 5 | (A) Post-stimulus time histogram of neural response evoked by INS stimulation (gray bar). (B,C) Increased INS radiant exposure leads to an increase in intrinsic signal magnitude. (B) Time course of signal for different radiant exposures. (C) Radiant exposure vs. peak amplitude of the intrinsic signal. Relationship fit with a linear equation. (Laser parameters: $\lambda=1.875 \mu \mathrm{m}, 250 \mu \mathrm{s}$ pulses at $200 \mathrm{~Hz}$ for $500 \mathrm{~ms}, 400 \mu \mathrm{m}$ fiber; D,E) INS potentiates response to visual stimulation. (D) Red and blue outlines demarcate left and right: ocular dominance columns, respectively. Left: OD map generated by subtraction of left minus right eye. Right: INS applied to left eye column during visual stimulation leads to relative enhancement of left eye columns (darkening in red outlined areas) and relative suppression of right eye columns (lightening in blue outlined areas). Scale bar: $1 \mathrm{~mm}$. (E) This is observed by examining averaged time courses from pixels in left eye (left panel, red arrow indicates increase in optical signal reflectance) and right eye (right panel, red arrow indicates decline in optical signal reflectance) columns. Dark gray bar: INS stimulation period. Light gray bar: visual stimulation period. Error bars: SEM. (INS parameters: $\lambda=1.875 \mu \mathrm{m}, 1.3 \mathrm{~J} / \mathrm{cm}^{2}, 250 \mu \mathrm{s}$ pulses at $200 \mathrm{~Hz}$ for $500 \mathrm{~ms}$, $100 \mu \mathrm{m}$ fiber). $Y$ axis: dR/R. (F) Effect of fiber size. Left: Schematic displaying 100, 200, and $400 \mu \mathrm{m}$ diameter fibers. Predicted intrinsic signal responses: $200 \mu \mathrm{m}$ fiber (red) produces larger enhancement than $100 \mu \mathrm{m}$ fiber (blue). However, $400 \mu \mathrm{m}$ fiber impinges on domains of other eye and leads to relative suppression (green). Right: actual data is consistent with prediction. (A-C from Cayce et al., 2011, D-F from Cayce et al., 2013).

INS stimulation to selected sites. As predicted, the induced percept of a light spot led to reliable eye saccades to the predicted site with the appropriate $300 \mathrm{~ms}$ latency for eye movements (Roe et al., 2013). This simple baseline test suggests that INS can be used to modulate behavioral outcome in ways similar to previous electrical stimulation studies. We predict that targeting stimulation to specific columnar locations will enable more selective behavioral effects than larger, multi-columnar activations.

\section{Optogenetic Stimulation}

The widespread use of optogenetics in neuroscience owes its thanks to the pioneering work of Karl Diesseroth, Edward Boyden, and their colleagues at Stanford and MIT. The key advantage of optogenetics is the ability to selectively target specific cell types. Neuronal response to optical stimulation is achieved by incorporation of light-sensitive rhodopsin molecules via molecular genetic techniques. They can be triggered with millisecond precision (Han and Boyden, 2007) and can lead to either excitatory (Boyden et al., 2005) or suppressive neuronal effects (Chow et al., 2010). Channelrhodopsin-2 (ChR2) is a light sensitive cation channel that leads to depolarization of neurons. Hyperpolarization can be achieved using halorhodopsin (a light-activated chloride pump) or archaerhodopsin (Arch; a light-activated proton pump; Boyden et al., 2005; Han and Boyden, 2007). In mice, this has been a boon to circuit dissection underlying behavior and disease (for review see Bernstein and Boyden, 2011). 

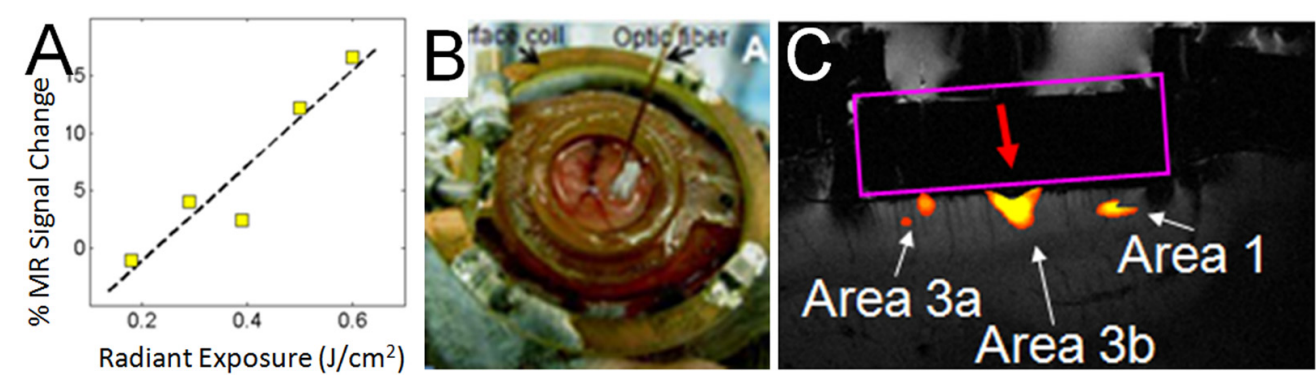

FIGURE 6 | Functional tract tracing with INS stimulation and fMRI. (A) Increasing INS stimulation intensity produces increasing BOLD response. (B) View of $400 \mu \mathrm{m}$ fiber optic applied to optical window at D2 location (as determined by optical imaging in different session) in area 3b of somatosensory cortex in a squirrel monkey. The monkey was imaged at 9.4T (Varian Inova Magnet) with a $3 \mathrm{~cm}$ surface coil surrounding the optical window. (C) Red arrow indicates the location of apposed optical fiber. INS stimulation $\left(0.4 \mathrm{~J} / \mathrm{cm}^{2}\right.$, pulsed trains applied in blocks of $30 \mathrm{~s}$ ON, $30 \mathrm{~s}$ OFF) at this site produces strong BOLD response at fiber tip as well as at corresponding topographic locations in area 3a and area 1. Coronal slice: voxel $625 \mu \mathrm{m} \times 625 \mu \mathrm{m} \times 1000 \mu \mathrm{m}$.

In monkeys, however, progress has been slower. Initial studies in primates demonstrated feasibility of expression and behavioral effect (Han et al., 2009, 2011; Diester et al., 2011; Cavanaugh et al., 2012; Galvan et al., 2012; Jazayeri et al., 2012). Surprisingly, unlike electrical stimulation, optogenetic stimulation appears to have more subtle effects. Rather than eliciting robust motor responses, optogenetic activation appears to modulate activity, either ongoing neural activity or to activity induced by electrical stimulation. Examples include optogenetic inactivation of monkey superior colliculus which leads to saccadic eye movement deficits (Cavanaugh et al., 2012), optogenetic activation in FEF which has subthreshold eye movement effects when paired with electrical stimulation (Ohayon et al., 2013), and optogenetic activation in parietal cortex which lead to modulation of salience maps (Dai et al., 2014). There may be multiple reasons for the apparent 'weaker' effect of optogenetic stimulation, such as differences in the mechanism of activation, differences in neural populations being activated, or the small number of cells effectively transfected.

Yet another potential cause relates to limitations in methodology. These limitations include inconsistencies in viral delivery, robustness of viral infection and expression, accuracy of light delivery, and reliability of in vivo assessments of neural or behavioral effectiveness. One approach for improving optogenetic technology in monkeys is the use of optical windows. In the primate, the presence of heavy dura mater has necessitated use of large bore injectrodes, electrodes, and optrodes (e.g., Diester et al., 2011) which can damage cortex; the opacity of dura mater also leads to uncertainty about injected location, targeting accuracy of optrodes, and inability to assess the degree of genetic expression. To alleviate some of these problems, the use of optical windows has permitted clear visualization and targeting of functionally characterized sites, use of fine injection pipettes which do not damage cortical tissue, and repeated optical monitoring of expression. These benefits of optical windows apply for optogenetics studies in both anesthetized and awake animals (Ruiz et al., 2013). Using optical windows, Nassi et al. (2015) have demonstrated that optogenetic stimulation in awake monkey visual cortex can 'substitute' for normal visual stimulation in a manner following the divisive normalization model of visual neuronal response and that neuronal response can be parametrically modulated in a controlled fashion. This important advance improves the reliability and accuracy of optogenetics methodology in primates and further secures its role as a focal stimulation modality in non-human primate studies.

\section{Functional Tract Tracing with Optogenetic Stimulation}

One of the most exciting applications of optogenetics is its use as a behavioral modulation tool and for functional tract tracing. In monkeys, this approach has been used to reveal frontal circuits underlying eye movement behavior. By stimulating ChR2 sites in FEF (the anterior and posterior arcuate sulcus) during performance of target selected eye saccades, Vanduffel and colleagues demonstrated that saccades in trials with stimulation occurred with significantly shorter latency than in those without (Gerits et al., 2012). The shortened saccade latencies suggested that optogenetic stimulation either produced greater neuronal firing rates or recruited a greater number of neurons. The success of this stimulation paradigm was attributed to a few factors: the use of a cell type non-specific CAG promoter, targeted injections in saccade related domains identified with FMRI, and stronger stimulation via the use of dual light guides. Importantly, the assessment of effect was not the induction of eye movement per se but rather the facilitation of eye movement latency. Thus, in contrast to early negative results, more finely tuned assessments of behavioral effect can reveal clear, robust effects of optogenetic stimulation. A further and important benefit of this optogenetic approach is its compatibility with the MRI environment. Stimulation of the anterior arcuate in the MR activated loci in V1, V4, MSTd, and MSTv, leading to identification of circuits underlying the speeded eye movement behavior.

At a local scale, optogenetic stimulation has also been used to probe the effect of stimulating single or multiple orientation domains in V1 of the tree shrew (Huang et al., 2014). Rather than use optrodes or fiber optics, this study used a spatial light modulator to produce a pre-specified pattern of blue light to activate specific ChR2 infected orientation columns in the 
cortex. Surprisingly, they did not find activation of nearby orientation domains of similar selectivity, but rather the effects appeared to fall off with distance from the stimulated domain. This result is not consistent with other results that demonstrate functionally selective effects of optogenetic stimulation via fiber optic delivery in Macaque monkey V1 (Chernov and Roe, 2014b). Stimulation of ocular dominance columns at single sites enhanced (increased reflectance change detected by optical imaging) the stimulated ocular dominance columns (similar to results obtained with INS, Figures 5D-F), and stimulation of single orientation domains led to relative enhancement of other orientation domains of similar selectivity. The differences between these studies may relate to intensity or effective depth of stimulation. Further studies are needed to evaluate these effects.

\section{CONCLUSION}

We have explored three different functional tract tracing methods that can be presented at the columnar scale in a targeted, functionally specific fashion. These methods do not require animal sacrifice and lengthy anatomical reconstruction, and potentially permit the study of connections from a greater number of sites within an individual animal. The similarities and differences between these methods and traditional anatomical tract tracing methods (e.g., in sensitivity to less robust connections) need to be further characterized and understood. While these methods cannot replace the gold standard of anatomical tract tracing, the advantages of in vivo functional tract tracing will make studying column-specific brain connections on a large scale more feasible.

The three methods presented each have their strengths and weaknesses. Electrical microstimulation is complicated by current spread but, at least within certain parameters and at a population level, appears to bias the activated circuits in a way that permits visualization of connections via functional imaging methods. Given that there is a wealth of studies demonstrating behavioral modulation using electrical stimulation, the use of

\section{REFERENCES}

Arsenault, J. T., Rima, S., Stemmann, H., and Vanduffel, W. (2014). Role of the primate ventral tegmental area in reinforcement and motivation. Curr. Biol. 24, 1347-1353. doi: 10.1016/j.cub.2014.04.044

Baek, K., Shim, W. H., Jeong, J., Radhakrishnan, H., Rosen, B. R., Boas, D., et al. (2015). Layer-specific interhemispheric functional connectivity in the somatosensory cortex of rats: resting state electrophysiology and fMRI studies. Brain Struct. Funct. [Epub ahead of print].

Bernstein, J. G., and Boyden, E. S. (2011). Optogenetic tools for analyzing the neural circuits of behavior. Trends Cogn. Sci. 15, 592-600. doi: 10.1016/j.tics.2011.10.003

Bosking, W. H., Zhang, Y., Schofield, B., and Fitzpatrick, D. (1997). Orientation selectivity and the arrangement of horizontal connections in tree shrew striate cortex. J. Neurosci. 17, 2112-2127.

Boyden, E. S., Zhang, F., Bamberg, E., Nagel, G., and Deisseroth, K. (2005). Millisecond-timescale, genetically targeted optical control of neural activity. Nat. Neurosci. 8, 1263-1268. doi: 10.1038/n n1525 electrical stimulation to map connections in the brain provides a direct link to these studies and could be conducted via methods that are already readily accessible. INS stimulation is a new method that is being developed for superficial and deep tissue stimulation. Applied via fiber optics, INS permits focal stimulation. INS does not require the use of viruses, giving it greater potential for human use. However, at this point in time, INS requires the delivery of specific infrared light wavelengths that are not yet commercially available. Optogenetics is a cell specific stimulation method that has revolutionized neuroscience. It requires the introduction of viruses that express light-sensitive rhodopsin molecules. Optogenetics has been enormously useful in studies in mice; its use in monkeys now also looks promising. Given the need for viral transfection, it is less amenable to use in humans.

The promise of conducting in vivo functional tract tracing studies calls for new future studies. We propose: (1) targeted, systematic, and large scale evaluation of columnar networks in different functional brain systems in monkeys (e.g., visual system, hand use system, working memory system), (2) cell type specific columnar mapping, (3) direct correlation of behavioral effects and mapped columnar networks, and (4) designing brainmachine interfaces based on targeting of columnar networks.

\section{ACKNOWLEDGMENTS}

We thank Duco Jansen and Anita Mahadevan-Jansen for their collaboration in the INS projects and the use of the INS laser (Capella Infrared Neuro-Stimulator, Lockheed Martin Aculight, Bothell WA). We thank John Gore and the Vanderbilt University Institute of Imaging Sciences for fMRI imaging support. We thank NIH for funding support: NS044375 (NINDS), MH095009 (NIMH), EY022853 (NEI), NS079471 (NINDS), as well as support from Vanderbilt Vision Research Center and the Center for Integrative and Cognitive Neuroscience at Vanderbilt. We also thank Zhejiang University for their support during the writing of this manuscript.

Brock, A. A., Friedman, R. M., Fan, R. H., and Roe, A. W. (2013). Optical imaging of cortical networks via intracortical microstimulation. J. Neurophysiol. 110, 2670-2678. doi: 10.1152/jn.00879.2012

Bullmore, E., and Sporns, O. (2009). Complex brain networks: graph theoretical analysis of structural and functional systems. Nat. Rev. Neurosci. 10, 186-198. doi: $10.1038 / \mathrm{nrn} 2575$

Cavanaugh, J., Monosov, I. E., McAlonan, K., Berman, R., Smith, M. K., Cao, V., et al. (2012). Optogenetic inactivation modifies monkey visuomotor behavior. Neuron 76, 901-907. doi: 10.1016/j.neuron.2012.10.016

Cayce, J., Friedman, R. M., Jansen, D., Mahavaden-Jansen, A., and Roe, A. W. (2011). Pulsed infrared light alters neural activity in rat somatosensory cortex in vivo. Neuroimage 57, 155-166. doi: 10.1016/j.neuroimage.2011.03.084

Cayce, J., Friedman, R. M., Jansen, D., Mahadevan-Jansen, A., and Roe, A. W. (2013). Infrared neural stimulation of primary visual cortex in non-human primates. Neuroimage 84, 181-190. doi: 10.1016/j.neuroimage.2013.08.040

Chase, S. M., Kass, R. E., and Schwartz, A. B. (2012). Behavioral and neural correlates of visuomotor adaptation observed through a brain-computer interface in primary motor cortex. J. Neurophysiol. 108, 624-644. doi: 10.1152/jn.00371.2011 
Chen, G., Wang, F., Dillenburger, C. D., Friedman, R. M., Chen, L. M., Gore, J. C., et al. (2012a). Functional magnetic resonance imaging of awake monkeys: some approaches for improving imaging quality. Magn. Reson. Imaging 30, 36-47. doi: 10.1016/j.mri.2011.09.010

Chen, G., Wang, F., Gore, J. C., and Roe, A. W. (2012b). Identification of cortical lamination in awake monkeys by high resolution magnetic resonance imaging. Neuroimage 59, 3441-3449. doi: 10.1016/j.neuroimage.2011.10.079

Chen, G., Cayce, J. M., Friedman, R. M., Wang, F., Tang, C., Jansen, D. E., et al. (2012c). Functional tract tracing in non-human primates using pulsed infrared lasers in conjunction with optical imaging and fMRI. Paper Presented at the Society for Neuroscience Meeting, New Orleans, LA.

Chen, G. C., Wang, F., Gore, J. C., and Roe, A. W. (2013). Layer-specific BOLD activation in awake monkey $\mathrm{V} 1$ revealed by ultra-high spatial resolution functional magnetic resonance imaging. Neuroimage 64, 147-155. doi: 10.1016/j.neuroimage.2012.08.060

Chen, L. M., Turner, G., Friedman, R. M., Gore, J. C., Roe, A. W., and Avison, M. J. (2007). High resolution maps of real and illusory tactile activation in SI: intra-individual correlation with fMRI, optical imaging and electrophysiology. J. Neurosci. 27, 9181-9191. doi: 10.1523/JNEUROSCI.1588-07.2007

Cheng, K., Waggoner, R. A., and Tanaka, K. (2001). Human ocular dominance columns as revealed by high-field functional magnetic resonance imaging. Neuron 32, 359-374. doi: 10.1016/S0896-6273(01)00477-9

Chernov, M., and Roe, A. W. (2014a). Infrared neural stimulation: a new stimulation tool for CNS applications. Neurophotonics 1:011011 doi: 10.1117/1.NPh.1.1.011011

Chernov, M., and Roe, A. W. (2014b). Optogenetic stimulation of monkey primary visual cortex. Paper Presented at the Society for Neuroscience Meeting, Washington, DC.

Chow, B. Y., Han, X., Dobry, A. S., Qian, X., Chuong, A. S., Li, M., et al. (2010). High-performance genetically targetable optical neural silencing by light-driven proton pumps. Nature 463, 98-102. doi: 10.1038/nature08652

Cooke, D. F., Taylor, C. S., Moore, T., and Graziano, M. S. (2003). Complex movements evoked by microstimulation of the ventral intraparietal area. Proc. Natl. Acad. Sci. U.S.A. 100, 6163-6168. doi: 10.1073/pnas.1031751100

Dai, J., Brooks, D. I., and Sheinberg, D. L. (2014). Optogenetic and electrical microstimulation systematically bias visuospatial choice in primates. Curr. Biol. 24, 63-69. doi: 10.1016/j.cub.2013.11.011

Diester, I., Kaufman, M. T., Mogri, M., Pashaie, R., Goo, W., Yizhar, O., et al. (2011). Anoptogenetic toolbox designed for primates. Nat. Neurosci. 14, 387-397. doi: 10.1038/nn.2749

Driver, J., Blankenburg, F., Bestmann, S., and Ruff, C. C. (2010). New approaches to the study of human brain networks underlying spatial attention and related processes. Exp. Brain Res. 206, 153-162. doi: 10.1007/s00221-010-2205-7

Ekstrom, L. B., Roelfsema, P. R., Arsenault, J. T., Bonmassar, G., and Vanduffel, W. (2008). Bottom-up dependent gating of frontal signals in early visual cortex. Science 321, 414-417. doi: 10.1126/science.1153276

Ekstrom, L. B., Roelfsema, P. R., Arsenault, J. T., Kolster, H., and Vanduffel, W. (2009). Modulation of the contrast response function by electrical microstimulation of the macaque frontal eye field. J. Neurosci. 29, 10683-10694. doi: 10.1523/JNEUROSCI.0673-09.2009

Federer, F., Williams, D., Ichida, J. M., Merlin, S., and Angelucci, A. (2013). Two projection streams from macaque $\mathrm{V} 1$ to the pale cytochrome oxidase stripes of V2. J. Neurosci. 33, 11530-11539. doi: 10.1523/JNEUROSCI.5053-12.2013

Galvan, A., Hu, X., Smith, Y., and Wichmann, T. (2012). In vivo optogenetic control of striatal and thalamic neurons in non-human primates. PLoS ONE 7:e50808. doi: 10.1371/journal.pone.0050808

Gerits, A., Farivar, R., Rosen, B. R., Wald, L. L., Boyden, E. S., and Vanduffel, W. (2012). Optogenetically induced behavioral and functional network changes in primates. Curr. Biol. 22, 1722-1726. doi: 10.1016/j.cub.2012.07.023

Godde, B., Leonhardt, R., Cords, S. M., and DInse, H. R. (2002). Plasticity of orientation preference maps in the visual cortex of adult cats. Proc. Natl. Acad. Sci. U.S.A. 99, 6352-6357.

Goense, J., Merkle, H., and Logothetis, N. K. (2012). High-resolution fMRI reveals laminar differences in neurovascular coupling between positive and negative BOLD responses. Neuron 76, 629-639. doi: 10.1016/j.neuron.2012. 09.019
Graziano, M. S., Taylor, C. S., and Moore, T. (2002). Complex movements evoked by microstimulation of precentral cortex. Neuron 34, 841-851. doi: 10.1016/S0896-6273(02)00698-0

Han, X., and Boyden, E. S. (2007). Multiple-color optical activation, silencing, and desynchronization of neural activity, with single-spike temporal resolution. PLoS ONE 2:e299. doi: 10.1371/journal.pone.0000299

Han, X., Chow, B. Y., Zhou, H., Klapoetke, N. C., Chuong, A., Rajimehr, R., et al. (2011). A high-light sensitivity optical neural silencer: development and application to optogenetic control of non-human primate cortex. Front. Syst. Neurosci. 5:18. doi: 10.3389/fnsys.2011.00018

Han, X., Qian, X., Bernstein, J. G., Zhou, H. H., Franzesi, G. T., Stern, P., et al. (2009). Millisecond-timescale optical control of neural dynamics in the nonhuman primate brain. Neuron 62, 191-198. doi: 10.1016/j.neuron.2009.03.011

Histed, M. H., Bonin, V., and Reid, R. C. (2009). Direct activation of sparse, distributed population of cortical neurons by electrical microstimulation. Neuron 63, 508-522. doi: 10.1016/j.neuron.2009.07.016

Huang, X., Elyada, Y. M., Bosking, W. H., Walker, T., and Fitzpatrick, D. (2014). Optogenetic assessment of horizontal interactions in primary visual cortex. J. Neurosci. 34, 4976-4990. doi: 10.1523/JNEUROSCI.4116-13.2014

Jazayeri, M., Lindbloom-Brown, Z., and Horwitz, G. D. (2012). Saccadic eye movements evoked by optogenetic activation of primate V1. Nat. Neurosci. 15, 1368-1370. doi: 10.1038/nn.3210

Jbabdi, S., Lehman, J. F., Haber, S. N., and Behrens, T. E. (2013). Human and monkey ventral prefrontal fibers use the same organizational principles to reach their targets: tracing versus tractography. J. Neurosci. 33, 3190-3201. doi: 10.1523/JNEUROSCI.2457-12.2013

Johnen, V. M., Neubert, F. X., Buch, E. R., Verhagen, L., O’Reilly, J. X., Mars, R. B., et al. (2015). Causal manipulation of functional connectivity in a specific neural pathway during behaviour and at rest. Elife 9:4. doi: 10.7554/eLife. 04585

Krieg, T. D., Salinas, F. S., Narayana, S., Fox, P. T., and Mogul, D. J. (2013). PET-based confirmation of orientation sensitivity of TMS-induced cortical activation in humans. Brain Stimul. 6, 898-904. doi: 10.1016/j.brs.2013.05.007

Livingstone, M. S., and Hubel, D. H. (1984). Anatomy and physiology of a color system in the primate visual cortex. J. Neurosci. 4, 309-356.

Lu, H. D., and Roe, A. W. (2008). Functional organization of color domains in V1 and V2 of Macaque monkey revealed by optical imaging. Cereb. Cortex 18, 516-533. doi: 10.1093/cercor/bhm081

Lujan, J. L., Chaturvedi, A., Choi, K. S., Holtzheimer, P. E., Gross, R. E., Mayberg, H. S., et al. (2013). Tractography-activation models applied to subcallosal cingulate deep brain stimulation. Brain Stimul. 6, 737-739. doi: 10.1016/j.brs.2013.03.008

Malach, R., Amir, Y., Harel, M., and Grinvald, A. (1993). Relationship between intrinsic connections and functional architecture revealed by optical imaging and in vivo targeted biocytin injections in primate striate cortex. Proc. Natl. Acad. Sci. U.S.A. 90, 10469-10473. doi: 10.1073/pnas.90.22.10469

Metin, C., Godement, P., and Imbert, M. (1988). The primary visual cortex in the mouse: receptive field properties and functional organization. Exp. Brain Res. 69, 594-612. doi: 10.1007/BF00247312

Moeller, S., Freiwald, W. A., and Tsao, D. Y. (2008). Patches with links: a unified system for processing faces in the macaque temporal lobe. Science 320, 13551359. doi: $10.1126 /$ science. 1157436

Murphey, D. K., and Maunsell, J. H. R. (2007). Behavioral detection of electrical microstimulation in different cortical visual areas. Curr. Biol. 17, 862-867. doi: 10.1016/j.cub.2007.03.066

Nassi, J. J., Avery, M. C., Cetin, A. H., Roe, A. W., and Reynolds, J. H. (2015). Normalization through local excitation and inhibition in primate visual cortex. Neuron 86, 1504-1517. doi: 10.1016/j.neuron.2015.05.040

Negyessy, L., Palfi, E., Ashaber, M., Palmer, C., Balazs, J., Friedman, R. M., et al. (2013). Intrinsic horizontal connections process global haptic features in the primary somatosensory cortex: neuroanatomical evidence. J. Comp. Neurol. 521, 2798-2817. doi: 10.1002/cne.23317

Ohayon, S., Grimaldi, P., Schweers, N., and Tsao, D. Y. (2013). Saccade modulation by optical and electrical stimulation in the macaque frontal eye field. J. Neurosci. 33, 16684-16697. doi: 10.1523/JNEUROSCI.2675-13.2013 
Olman, C. A., Harel, N., Feinberg, D. A., He, S., Zhang, P., Ugurbil, K., et al. (2012). Layer-specific fMRI reflects different neuronal computations at different depths in human V1. PLoS ONE 7:e32536. doi: 10.1371/journal.pone.0032536

Raichle, M. E. (2015). The restless brain: how intrinsic activity organizes brain function. Philos. Trans. R. Soc. Lond. B Biol. Sci. 370. doi: 10.1098/rstb.2014.0172

Rasmussen, T., and Penfield, W. (1947). The human sensorimotor cortex as studied by electrical stimulation. Fed. Proc. 6(1Pt. 2), 184.

Rockland, K. S. (2010). Five points on columns. Front. Neuroanat. 4:22. doi: $10.3389 /$ fnana.2010.00022

Roe, A. W., Chen, G., Cayce, J. M., Ye, X., Jansen, E. D., and Mahadevan-Jansen, A. (2013). Infrared neural stimulation of visual cortex induces visual perception in awake non-human primates. Paper Presented at the Society for Neuroscience Meeting, San Diego, CA.

Roe, A. W., and Ts'o, D. Y. (1999). Specificity of color connectivity between primate V1 and V2. J. Neurophysiol. 82, 2719-2731.

Roe, A. W., and Ts'o, D. Y. (2015). Specificity of V1-V2 orientation networks in the primate visual cortex. Cortex doi: 10.1016/j.cortex.2015.07.007 [Epub ahead of print].

Romo, R., Hernandez, A., Zainos, A., Brody, C. D., and Lemus, L. (2000). Sensing without touching: psychophysical performance based on cortical microstimulation. Neuron 26, 273-278. doi: 10.1016/S0896-6273(00)81 156-3

Ruff, C. C., Driver, J., and Bestmann, S. (2009). Combining TMS and fMRI: from 'virtual lesions' to functional-network accounts of cognition. Cortex 45, 1043-1049. doi: 10.1016/j.cortex.2008.10.012

Ruiz, O., Lustig, B., Nassi, J. J., Reynolds, J., Callaway, E., Albright, T., et al. (2013). Optogenetics through windows on the brain in the nonhuman primate. J. Neurophysiol. 110, 1455-1467. doi: 10.1152/jn.00153.2013

Salzman, C. D., Britten, K. H., and Newsome, W. T. (1990). Cortical microstimulation influences perceptual judgements of motion direction. Nature 346, 174-177. doi: 10.1038/346174a0

Schuett, S., Bonhoeffer, T., and Hübener, M. (2002). Mapping retinotopic structure in mouse visual cortex with optical imaging. J. Neurosci. 22, 6549-6559.

Shapiro, M. G., Homma, K., Villarreal, S., Richter, C.-P., and Bezanilla, F. (2012). Infrared light excites cells by changing their electrical capacitance. Nat. Commun. 3, 736. doi: 10.1038/ncomms 1742

Shih, Y. Y., Chen, Y. Y., Lai, H. Y., Kao, Y. C., Shyu, B. C., and Duong, T. Q. (2013). Ultra high-resolution fMRI and electrophysiology of the rat primary somatosensory cortex. Neuroimage 73, 113-120. doi: 10.1016/j.n euroimage.2013.01.062

Shmuel, A., Korman, M., Sterkin, A., Harel, M., Ullman, S., Malach, R., et al. (2005). Retinotopic axis specificity and selective clustering of feedback projections from V2 to V1 in the owl monkey. J. Neurosci. 25, 2117-2131. doi: 10.1523/JNEUROSCI.4137-04.2005

Sincich, L. C., and Blasdel, G. G. (2001). Oriented axon projections in primary visual cortex of the monkey. J. Neurosci. 21, 4416-4426.

Sincich, L. C., Jocson, C. M., and Horton, J. C. (2010). V1 interpatch projections to V2 thick stripes and pale stripes. J. Neurosci. 30, 6963-6974. doi: 10.1523/JNEUROSCI.5506-09.2010

Sporns, O. (2010). Networks of the Brain. Cambridge, MA: MIT Press.

Stepniewska, I., Cerkevich, C. M., and Kaas, J. H. (2015). Cortical connections of the caudal portion of posterior parietal cortex in prosimian Galagos. Cereb. Cortex doi: 10.1093/cercor/bhv132 [Epub ahead of print].

Stepniewska, I., Fang, P. Y., and Kaas, J. H. (2009). Organization of the posterior parietal cortex in galagos: functional zones identified by microstimulation. J. Comp. Neurol. 517, 765-782. doi: 10.1002/cne.22181

Stepniewska, I., Friedman, R. M., Gharbawie, O. A., Cerkevich, C. M., Roe, A. W., and Kaas, J. H. (2011). Optical imaging in galagos reveals parietalfrontal circuits underlying motor behavior. Proc. Natl. Acad. Sci. U.S.A. 108, E725-E732. doi: 10.1073/pnas.1109925108

Sun, P., Gardner, J. L., Costagli, M., Ueno, K., Waggoner, R. A., Tanaka, K., et al. (2013). Demonstration of tuning to stimulus orientation in the human visual cortex: a high-resolution fMRI study with a novel continuous and periodic stimulation paradigm. Cereb. Cortex 23, 1618-1629. doi: 10.1093/cercor/ bhs149

Tanigawa, H., Lu, H. D., and Roe, A. W. (2010). Functional organization for color and orientation in macaque V4. Nat. Neurosci. 13, 1542-1548. doi: 10.1038/nn.2676

Tehovnik, E. J., and Slocum, W. M. (2009). Background luminance affects the detection of microampere currents delivered to macaque striate cortex. Eur. J. Neurosci. 30, 263-271. doi: 10.1111/j.1460-9568.2009.06810.x

Tehovnik, E. J., Slocum, W. M., and Schiller, P. H. (2004). Microstimulation of V1 delays the execution of visually guided saccades. Eur. J. Neurosci. 20, 264-272. doi: 10.1111/j.1460-9568.2004.03480.x

Tehovnik, E. J., Slocum, W. M., Smirnakis, S. M., and Tolias, A. S. (2009). Microstimulation of visual cortex to restore vision. Prog. Brain Res. 175, 347-375. doi: 10.1016/S0079-6123(09)17524-6

Tehovnik, E. J., Tolias, A. S., Sultan, F., Slocum, W. M., and Logothetis, N. K. (2006). Direct and indirect activation of cortical neurons by electrical microstimulation. J. Neurophysiol. 96, 512-521. doi: 10.1152/jn.00126.2006

Thomas, C., Ye, F. Q., Irfanoglu, M. O., Modi, P., Saleem, K. S., Leopold, D. A., et al. (2014). Anatomical accuracy of brain connections derived from diffusion MRI tractography is inherently limited. Proc. Natl. Acad. Sci. U.S.A. 111, 16574-16579. doi: 10.1073/pnas.1405672111

Tolias, A. S., Sultan, F., Augath, M., Oeltermann, A., Tehovnik, E. J., Schiller, P. H., et al. (2005). Mapping cortical activity elicited with electrical microstimulation using fMRI in the macaque. Neuron 48, 901-911. doi: 10.1016/j.neuron.2005.11.034

Ts'o, D. Y., Gilbert, C. D., and Wiesel, T. N. (1986). Relationships between horizontal interactions and functional architecture in cat striate cortex as revealed by cross-correlation analysis. J. Neurosci. 6, 1160-1170.

Wang, Z., Negyessy, L., Chen, L. M., Friedman, R. M., John G., and Roe, A. W. (2013). The relationship of anatomical and functional connectivity to resting state connectivity in primate somatosensory cortex. Neuron $78,1116-1126$. doi: 10.1016/j.neuron.2013.04.023

Wells, J., Kao, C., Jansen, E. D., Konrad, P., and Mahadevan-Jansen, A. (2005a). Application of infrared light for in vivo neural stimulation. J. Biomed. Opt. 10. doi: $10.1117 / 1.2121772$

Wells, J., Kao, C., Mariappan, K., Albea, J., Jansen, Konrad, P., et al. (2005b). Optical stimulation of neural tissue in vivo. Opt. Lett. 30, 504-506. doi: 10.1364/OL.30.000504

Wells, J., Kao, C., Konrad, P., Milner, T., Kim, J., Mahadevan-Jansen, A., et al. (2007a). Biophysical mechanisms of transient optical stimulation of peripheral nerve. Biophys. J. 93, 2567-2580.

Wells, J., Konrad, P., Kao, C., Jansen, and Mahadevan-Jansen, A. (2007b). Pulsed laser versus electrical energy for peripheral nerve stimulation. J. Neurosci. Methods 163, 326-337. doi: 10.1016/j.jneumeth.2007.03.016

Yacoub, E., Harel, N., and Ugurbil, K. (2008). High-field fMRI unveils orientation columns in humans. Proc. Natl. Acad. Sci. U.S.A. 105, 10607-10612. doi: 10.1073/pnas.0804110105

Yoshioka, T., Levitt, J. B., and Lund, J. S. (1992). Intrinsic lattice connections of macaque monkey visual cortical area V4. J. Neurosci. 12, 2785-2802.

Zingg, B., Hintiryan, H., Gou, L., Song, M. Y., Bay, M., Bienkowski, M. S., et al. (2014). Neural networks of the mouse neocortex. Cell 156, 1096-1111. doi: 10.1016/j.cell.2014.02.023

Conflict of Interest Statement: The authors declare that the research was conducted in the absence of any commercial or financial relationships that could be construed as a potential conflict of interest.

Copyright (c) 2015 Roe, Chernov, Friedman and Chen. This is an open-access article distributed under the terms of the Creative Commons Attribution License (CC BY). The use, distribution or reproduction in other forums is permitted, provided the original author(s) or licensor are credited and that the original publication in this journal is cited, in accordance with accepted academic practice. No use, distribution or reproduction is permitted which does not comply with these terms. 